\title{
Propiedades psicométricas de la escala de bienestar PERM A para adolescentes: alternativas para su medición Psychometric properties of the PERM A well-being scale for adolescents: alternatives for its measurement
}

\author{
*Diego García-Álvarez, **Juan Hernández-Lalinde, ***M aría José Soler, Rubia Cobo-Rendón, \\ ** hon-Franklin Espinosa-Castro \\ *Universidad Rafael Belloso Chacín (Venezuela), **Universidad Simón Bolívar (Colombia), ***Asociación Civil Jóvenes \\ Fuertes (Uruguay), ****U niversidad Santo Tomás (Chile)
}

\begin{abstract}
Resumen. La adolescencia es una etapa del ciclo vital que implica grandes cambios en todas las facetas de la vida. En dicha etapa, la persona está expuestaa múl tiples factores de riesgo y de protección que pueden incidir en su salud, por lo que estudiar el constructo del bienestar se hace necesario para comprender a mayor profundidad la salud mental en este periodo. A pesar delo anterior, se ha obser vado en el contexto latinoamericano una ausenciaimportante de instrumentos disenados paratal fin, de modo que el objetivo de esta investigación fue evaluar las propiedades psicométricas de la escala de bienestar psicológico adolescente diseñada por Soler, sustentada en la teoría PERM A de Seligman y presentar alternativas a la estructura factorial original. Se realizó una investigación instrumental en la que participaron 779 adolescentes de M ontevideo, muestra que fue dividida al eatoriamente en dos partes para efectuar una validación cruzada. En la muestra de entrenamiento $(n=390)$ se llevó a cabo un análisis factorial exploratorio que reveló opciones de cuatro y cinco dimensiones. Posteriormente, se evaluaron cuatro modelos en la muestra de prueba $(n=389)$ mediante un análisis factorial confirmatorio, destacándose dos estructuras pentadimensionales, la original (35 ítems) y la abreviada (20 ítems). En ambas se encontraron correlaciones positivas con bienestar, autoestima y autoeficacia, y negativas con síntomas depresivos y malestar psicológico; sin embargo, la validez convergente y discriminante del modelo abreviado fue superior. La consistencia interna de cada factor varió desde inaceptable hasta excelente, aunque en promedio puede considerarse adecuada. Se concluye que la escala formulada por Soler en su versión abreviada posee mejores propiedades psicométricas que la original, constituyéndose en un instrumento válido y confiable que puede ser administrado en la población adolescente montevideana para evaluar el bienestar psicológico multidimensional.
\end{abstract}

Palabras clave: bienestar psicológico, adolescentes, análisis factorial, estructura factorial, validación, M ontevideo.

\begin{abstract}
Adolescence is a stage in the life cycle that involves great changes in all facets of life. In this stage, the person is exposed to multiple risk and protective factors that can affect their health, so studying the construct of well-being is necessary to understand more deeply the mental heal th in this period. D espite the above, an important absence of instruments designed for this purpose has been observed in the Latin American context, so the aim of this research wasto evaluate the psychometric properties of the adolescent psychological well-being scale designed by Soler, based on Seligman's PERM A theory, and to present alternatives to the original factor structure. An instrumental investigation was carried out in which 779 adolescents from M ontevideo participated, a samplethat was randomly divided into two parts to carry out a cross-val idation. An exploratory factor analysis was carried out in the training sample $(n=390)$, which revealed four and fivedimensional options. Subsequently, four models were evaluated in the test sample $(n=389)$ by means of a confirmatory factor analysis, highlighting two pentadimensional structures, the original ( 35 items) and the abbreviated (20 items). In both, positive correlations were found with well-being, self-esteem and self-efficacy, and negative correlations with depressive symptoms and psychological distress; however, the convergent and discriminant validity of the abbreviated model was superior. The internal consistency of each factor ranged from unacceptable to excellent, although on average it can be consider ed adequate. It isconcluded that the scale formul ated by Soler in its abbreviated ver sion hasbetter psychometric properties than theoriginal one, constituting avalid and reliable instrument that can be administered in the M ontevidean adolescent population to assess psychological well-being Keywords: psychological well-being, adolescents, factor analysis, factor structure, validation, M ontevideo.
\end{abstract}

\section{Introducción}

Promover el bienestar es fundamental para que las personas puedan experimentar una vida con sentido, ser ciudadanos creativos, activos y comprometidos consigo mismos y con la sociedad (Isabel, 2016). A nivel mundial, la promoción del bienestar en adolescentes es un tema de gran importancia para la salud pública y social. La adolescencia es una etapa que implica grandes cam-

\footnotetext{
Fecha recepción: 05-08-20. Fecha de aceptación: 23-11-20

Juan Diego Hernández Lalinde

j.hernandezl@ unisimonbolivar.edu.co
}

bios en todas las facetas de la vida debido a las nuevas demandas ambientales que se le imprimen a la persona adolescente; a saber, mayor exigencia académica, cambios corporales, manejo de relaciones e incluso primeras experiencias de amor y otras más, por lo que estudiar el bienestar se hace necesario para comprender a mayor profundidad la salud mental en la adolescencia (De Carvalho, Pereira, Pinto \& Maroco, 2016; GarcíaÁlvarez, Hernández-Lalinde, Espinosa-Castro \& Soler, 2020; Sawyer et al., 2012).

La investigación del bienestar plantea dos enfoques parasu estudio, el subjetivo o hedónico y el eudaimónico 
(Braaten, Huta, Tyrany \& Thompson, 2019; Jongbloed, 2015). El bienestar subjetivo corresponde a la evalua ción de los afectos positivos, afectos negativos y de la satisfacción con la vida (Diener, 0 ishi \& Tay, 2018; Jayawickreme, Forgeard \& Seligman, 2012). En general, se define al bienestar psicológico como el grado en que las personas sienten que avanzan en su crecimiento personal y en su percepción acerca de «hacer el bien». Este se basa en los conceptos de relaciones positivas, significado y vida con propósito (Jayawickreme et al., 2012). La presencia de estas dos filosofías ha incrementado la imprecisión del concepto del bienestar, es por ello que hoy en día se consideran conjuntamente la aproximación hedónica y eudaimónica, para de esta manera tener una visión amplia de este constructo en la vida de las personas (Hone, Jarden, Schofield \& Duncan, 2014; Shinde, 2017).

El bienestar es considerado un constructo complejo y multifactorial (Pollard \& Lee, 2003). El mismo surge de una evaluación subjetiva que realiza la persona acerca de su existencia con una mirada retrospectiva; por tanto, la definición y medición científica del bienestar depende del enfoque teórico que brinde un camino para operacionalizarlo.

Históricamente, el bienestar subjetivo o hedónico se ha conceptualizado a partir del modelo teórico propuesto por Diener, Emmons, Larsen \& Griffin (1985). Según este modelo, el bienestar subjetivo se encuentra compuesto por tres componentes: la satisfacción con la vida, el afecto positivo y el afecto negativo (Disabato, Goodman, Kashdan, Short \& Jarden, 2016). Por otra parte, las investigaciones sobre el constructo de bienestar eudaimónico se han fundamentado en su mayoría desde la teoría propuesta por Ryff (1989), la cual describe que este tipo de bienestar se sustenta en la eva luación cognitiva que realizan las personas considerando los factores de autonomía, crecimiento personal, autoaceptación, propósito de vida, dominio del entorno y relaciones positivas con los demás (Ryff, 2016). En este caso, el bienestar eudaimónico representa la evaluación cognitiva de qué tanto la persona avanza en su desarrollo personal y en las acciones que contribuyen a ampliar su potencial a partir del equilibrio entre sus recursos individuales y los desafíos a los cuales se enfrenta (Disabato et al., 2016).

Seligman en 2011 propuso un modelo de cinco dimensiones interconectadas llamado PERM A. Este modelo define al bienestar como la búsqueda y alcance de los elementos que lo conforman, tal es como emociones positivas, compromiso, relaciones positivas, propósito y logros (Hone et al., 2014; Seligman, 2011). Estos autores argumentan que cada una de las dimensiones que integran esta teoría son intrínsecamente valiosas, por lo que las personas buscan experimentarlas. Estas dimensiones son valoradas como positivas y beneficiosas en sí mismas (Burke \& Minton, 2019). Además, se postula que todas pueden ser cuantificables de forma independiente, pero también de manera global para obtener una medida general de bienestar (Hone, 2015).

Las emociones positivas son consideradas como sentimientos hedónicos de felicidad que se asemejan al lado positivo del bienestar afectivo en términos de bienestar subjetivo. Por otro lado, las cuatro dimensiones restantes del modelo PERMA se relacionan con el bienestar psicológico desde unavisión eudaimónica(Kern, Waters, Adler $\&$ W hite, 2015). El compromiso se refiere a un estado mental positivo sustentado por la presencia de vigor, dedicación y absorción en las actividades que son de interés para las personas (Salanova, Schaufeli, Martinez \& Breso, 2010). Las relaciones positivas tienen que ver con la percepción que se tiene sobre la calidad y cantidad de estas (Seligman, 2011), las cuales se asocian con un mejor ajuste social (Morrish et al., 2018). El propósito se vincula con la noción de transcendencia mediante la promoción de las relaciones sociales o la conexión con un propósito definido (Schueller \& Seligman, 2010). Finalmente, la dimensión de logros se define como el desarrollo del potencial individual, implicando la persistencia a pesar de las dificultades para el logro de los objetivos personales (Norrish, W illiams, O 'Connor \& Robinson, 2013).

Desde el marco de la teoría PERMA, un estudio en adolescentes irlandeses identificó que el bienestar va disminuyendo a medida que aumentan los años escola res, pero también evidenció diferencias de acuerdo con el sexo teniendo los hombres mayores niveles de bienestar que las mujeres en cada una de sus dimensiones (Burke \& Minton, 2019). 0 tra investigación evaluó las implicaciones del bienestar en adolescentes durante la transición a la edad adulta. Los resultados encontrados señalaron que al tos niveles en las dimensiones que componen el PERMA se asociaron con indicadores de progreso profesional como logros educativos y competencia percibida, así como con la admisión de responsabilidades de ciudadanía tales como el voluntariado y las actividades cívicas ( 0 'C onnor, Sanson, Toumbourou, N orrish \& O Isson, 2017). Estudios recientes marcan la existencia de características en la adolescencia como el compromiso, la perseverancia, el optimismo, la interconexión y la felicidad que pueden contribuir a fortale- 
cer su bienestar en la vida adulta (Cobo-Rendón, Pérez, Hernández \& Aslan, 2017).

Adicionalmente, la evidencia empírica destaca el papel del bienestar en aspectos como la disminución de síntomas depresivos, en el desarrollo de la autoestima en adolescentes (M ateo, Hernández \& Cabrero, 2019; Sánchez-Hernández, Méndez, Ato \& Garber, 2019) y en la promoción de la autoeficacia académica, social y emocional, teniendo efectos positivos en la adquisición de habilidades para la vida (D emirtaô, 2020; Sagone, De Caroli, Falanga \& Indiana, 2020).

Una revisión sistemática mostró que hay pocas investigaciones sobre el modelo multidimensional de bienestar en niños y adolescentes, lo que podría ser un vacío en la conceptualización y promoción del bienestar a lo largo del ciclo vital (Cobo-Rendón et al., 2017). En términos de medición en adolescentes, otra revisión sistemática reciente identificó 11 escalas que buscan medir este constructo, cinco de las cual es fueron diseñadas en EstadosU nidos, en tanto que las otras fueron elaboradas en países como Dinamarca, Reino U nido, Escocia, India y Nueva Zelanda. O tro hallazgo importante reportado por los autores fue que, de los 11 instrumentos identificados, solo cuatro fueron propuestos original mente para su uso en adolescentes (Rose et al., 2017). Estos resultados muestran el poco desarrollo de escalas de medición en el área del bienestar psicológico en la etapa evolutiva adolescente, en especial en contextos latinoamericanos.

La investigación sostiene que los instrumentos planteados para adultos pueden no ser confiables y válidos entre los jóvenes debido a que su etapa de desarrollo es única en cuanto a las tareas psicosociales de la adolescencia (Rose et al., 2017). Por consiguiente, el presente trabajo se justifica desde la importancia que entraña la realización de procesos de validación que permitan asegurar la relevancia cultural y conceptual del bienestar en instrumentos para adolescentes, puesto que promover el bienestar en esta etapa es crítico para el campo de la práctica de la psicología clínica, educativa y social.

Debido a lo reciente de la teoría PERMA formula da por Seligman (2011), en la literatura científica se conocen pocas medidas psicométricas para medir este constructo de manera agrupada y considerando la totalidad de los cinco elementos; en efecto, Butler \& Kern (2016) desarrollaron la escala PERM A-Profiler con base en una muestra de 31966 adultos angloparlantes al rededor del mundo e integrada por un total de 15 ítems reflejando adecuadas propiedades psicométricas en confiabilidad, validez convergente y validez factorial al confirmar los cinco elementos del bienestar.

Sin embargo, cuando las autoras quisieron extender su modelo a la adolescencia tuvieron dificultades para validar la estructura factorial (Kern, Waters, W hite \& Adler, 2014) pues encontraron cuatro de los cinco factores; emociones positivas, compromiso, relaciones y logro, mientras que el elemento del sentido no se definió claramente, sino que saturó en el de relaciones. En vista de ello, formularon el modelo EPO CH (M easure of Adolescent Well-Being) como una integración entre el desarrollo positivo adolescente y el PERMA, entendiendo que esas características adolescentes influyen en los dominios de la estructura de Seligman en la adultez (Kern, Benson, Steinberg \& Steinberg, 2016).

Así pues, no se cuenta con una versión de la escala PERMA-Profiler para medir el bienestar adolescente. En contextos hispanoparlantes, Soler (2016) presentó en su tesis doctoral la construcción de una escal a formulada totalmente en español y basada en la teoría de bienestar PERM A en conjunto con las características psicológicas de la adolescencia que denominó «escal a de bienestar psicológico para adolescentes de Soler». En tal sentido, el objetivo del presente trabajo fue examinar las propiedades psicométricas de dicha escala, específicamente la validez factorial, convergente, discriminante, además de la confiabilidad en una muestra de adolescentes de M ontevideo, U ruguay.

\section{Metodología}

\section{Tipo de investigación}

Investigación instrumental (M ontero \& León, 2007) para establecer las propiedades psicométricas de escala de bienestar psicológico para adolescentes de Soler (2016).

\section{Participantes}

Participaron 779 adolescentes residenciados en la ciudad deM ontevideo, U ruguay. Todosfueron contactados en instituciones educativas, siendo escogidos mediante muestreo intencional. El tamaño de la muestra quedó definido por las limitaciones económicas, logísticas y de tiempo asociadas al proyecto. Los criterios de inclusión y exclusión fueron: (a) tener una edad comprendidaentre 11 y 19 años; (b) ser estudiante regular; (c) estar residenciados en la mencionada ciudad; y (d) carecer de condiciones físicas, mentales o emocionales que alterasen 0 impidiesen la participación en el estudio. 


\section{Instrumentos}

La escala de bienestar psicológico para adolescentes de Soler (2016) se basa en el modelo PERMA de Seligman (2011). Según este, el bienestar psicológico se explica a partir de las emociones positivas (positive emotions), compromiso (engagement), relaciones interpersonales(relationships), sentido devida(meaning) y metas (accomplishments). Esta medida se adapta a esta configuración y consta de 35 reactivos en estilo Likert. La autora presentó evidencias de validez de contenido por medio de la técnica de jueces con evaluación cualitativa de los ítems a nivel de redacción y pertinencia teórica. De igual manera, reportó evidencias de confiabilidad en emociones positivas (.71), compromiso $(.51)$, relaciones $(.67)$, sentido de vida $(.83)$ y metas (.75).

Ahora bien, en este estudio se emplearon otros instrumentos que sirvieron para establecer la validez convergente de la escala. En tal sentido, se administraron cuestionarios de autoestima (Rosenberg, 1965), autoeficacia (Schwarzer \& Baessler, 1996), bienestar psicológico (Casullo et al., 2002), síntomas depresivos (Herrero \& M eneses, 2006; Radloff, 1977) y malestar psicológico (Brenlla \& Aranguren, 2010; Kessler \& M roczek, 1994). Para profundizar, consúltese a O livaDelgado et al. (2011).

\section{Procedimiento}

En el inicio del proyecto se divulgaron los objetivos e implicaciones del estudio con los representantes de estudiantes menores de edad por medio de la junta directiva de las instituciones. Durante la etapa de aplica ción, se le explicó a la muestra de adolescentes el propósito, objetivo y se indicó que la información sería anónima y que se utilizaría solo para fines investigativos. Asimismo, se enfatizó en que la colaboración adolescente sería total mente voluntaria, que no estaría ligada a compensación alguna y que no entrañaba riesgos de ningún tipo. El consentimiento informado fue diligenciado con las instituciones educativas de Montevideo por medio de la junta directiva del plantel de acuerdo con los lineamientos éticos, normativos y lega les de la APA y del Ejecutivo uruguayo a través del Decreto 379/ 0081.

\section{Análisis estadístico}

En primer lugar, se verificó la normalidad y la ausencia de valores atípicos paralo cual se empleó la prueba de Mardia y las distancias de Mahalanobis. Estos procedimientos son los más recomendados cuando se evalúan tales suposiciones a nivel multivariado y se encuentran con frecuencia en las publicaciones científicas del área psicométrica (Trigueros, Aguilar-Parra, González-Santos \& Cangas, 2019). La normalidad fue rechazada y varios datos extremos fueron detectados. En segundo lugar, se encontró una fracción de datos perdidos en los ítems de los cuestionarios que alcanzó un máximo de $3.72 \%$ en el elemento 6 de la escala PERMA. En lo que respecta al número de reactivos sin responder por participante, se halló una proporción que no excedió de $5.71 \%$ en 15 de los 779 encuestados. Posteriormente, se aplicó la prueba de Little para determinar si la pérdidade información era completamenta al eatoria, supuesto que fue rechazado; por tanto, se completar on las ca sillas vacías mediante imputación múltiple, decisión que se sustentó en el porcentaje de valores ausentes. Aunque no hay una posición unánime, investigaciones sugieren fracciones no mayores de $5 \%$ ya que estas no generan consecuencias que afecten los resultados (Sainani, 2015).

A continuación, la muestra fue dividida aleatoriamente en dos partes, una constituida por 390 adolescentes que fue utilizada para el análisis factorial exploratorio (AFE) y la otra integrada por 389 participantes empleada en el análisis factorial confirmatorio (AFC). La utilización de ambas estrategias obedece no solo a la intención de comprobar la validez factorial de la versión original de la escala propuesta por Soler, sino también a la de proponer una adaptación a dicho instrumento (Díaz-Leal, Blanco-O rnelas, Benítez-Hernández, Aguirre-Vásquez \& Candia-Luján, 2017; DomínguezAlonso, López-Castedo \& PortelaPino, 2017). El AFE fue desarrollado mediante factores principales con determinante mínimo de covarianza y rotación oblimin, estableciendo previamente el número de variables la tentes. Se eliminaron los ítems cuyas cargas factoriales fueron menores que .40 o cuya comunalidad fue menor que .20. Los resultados de esta fase del trabajo, en conjunto con la teoría PERMA de bienestar psicológico, fueron elementos que sirvieron para el planteamiento de los modelos que serían evaluados en el AFC.

En lo concerniente al AFC, se eligió como método de estimación el de mínimos cuadrados ponderados con media y varianza ajustada (W LSMV), alternativa que ofrece ventajas cuando se manipulan variables ordinales caracterizadas por la ausencia de normalidad y la presencia de datos atípicos (Bandalos, 2014; Trigueros et al. , 2019). La adecuación de los modelos fue sopesada con la prueba chi-cuadrado y su respectivo nivel de significación, pero también mediante la relación entre este 
estadístico y los grados de libertad del procedimiento. Se utilizaron las medidas del error cuadrático medio por aproximación (RMSEA) y del residuo estandariza do cuadrático medio (SRMR), además de indicadores como el índice de bondad de ajuste (GFI), de bondad de ajuste corregido (AGFI), de ajuste comparativo (CLI) y de Tucker-Lewis (TLI). Estas medidas de ajuste son las sugeridas y las que se reportan en manuscritos como este (Díaz-Leal et al., 2017; Domínguez-Alonso et al., 2017; Trigueros et al., 2019).

La validez convergente fue establecida por medio de la varianza media extraída (VME) y a partir de las correlaciones de Spearman-Brown entre las puntuaciones de la escala y las obtenidas con los demás instrumentos. Al respecto, se utilizó este coeficiente en vista de que se violaron varios de los supuestos del coeficiente $R$ de Pearson (Hernández-Lalinde et al., 2018). Por otro lado, la validez discriminante se evalúo mediante la razón de las correlaciones HTMT (heterotraitmonotrait), criterio que ha mostrado un desempeño superior al de Fornell-Larcker y al de cargas factoriales cruzadas (Henseler et al., 2015). Las estimaciones puntuales de este procedimiento se obtuvieron con mínimos cuadrados parciales, en tanto que los errores estándares se derivaron a través de técnicas de bootstrapping con sesgo corregido y acelerado. Para la consistencia interna se calcularon los coeficientes alfa de Cronbach, omega de $\mathrm{M}$ CD onald e índice de fiabilidad compuesta (IFC), mientras que la homogeneidad se exploró a través de la correlación ítem-total corregida (CITC), utilizando nuevamente la alternativa no paramétrica de Spearman-Brown. En lo que respecta a los primeros índices, se obtuvieron los estimadores y el intervalo de confianza respectivo gracias al método de bootstrapping previamente mencionado; sin embargo, en el caso de la CITC, solo se reportaron la media, el mínimo y el máximo correspondientes a cada factor y al total.

Los datos fueron procesados y analizados con el programa SPSS en su versión 25 paraW indows de 64 bits y a través del lenguaje de programación estadístico R, específicamente de la aplicación R-Studio, utilizando los paquetes Lavaan, Semplots, SemTools, Mvn, M voutlier y Psych. La significación se determinó a partir de .05.

\section{Resultados}

\section{Características sociodemográficas de los parti- cipantes}

La muestra estuvo conformada por 779 adolescen- tes distribuidos en un $52.37 \%(n=408)$ de mujeres y en un $47.63 \%(n=371)$ de hombres. La edad varió desde 11 hasta 19 años, con media de $15.28 \pm 2.85$, en tanto quelos gruposetarios registraron porcentajes de $40.56 \%$ ( $n=316$ ) paraquienestenían entre 11 y 13 años, $16.56 \%$ $(n=129)$ paralos que reportaron desde 14 hasta 16 años, y $42.88 \%$ ( $n=334$ ) para aquellos con edad desde 17 hasta 19 años.

\section{Planteamiento de los modelos}

El planteamiento de los model os alternativos se desprendió delaproposición teórica de Seligman, pero también se apuntaló en los hallazgos del AFE aplicado a la muestra de entrenamiento $(n=390)$. La adecuación muestral y la presencia de factores subyacentesfue comprobada con la medida de Kaiser-M eyer-O Ikin ( $K M 0=.84)$ y la prueba de esfericidad de Barlett $\left(X^{2}=4334.60, g l=595, p<.001\right)$. Por su parte, la exploración realizada a través del gráfico de sedimentación sugirió la retención de seis factores, mientras que el análisis paralelo y la prueba de Velicer indicaron esquemas de cuatro y cinco dimensiones, respectivamente. En virtud de esto, se proyectaron tres nuevos modelos, cada uno conformado por cuatro, cinco y seis variables latentes.

Luego de esto, se procedió a realizar el AFE de cada propuesta. El criterio ya mencionado para la eliminación de los ítems llevó a que el modelo de cuatro factores estuviera compuesto por 21 reactivos, mientras que el de cinco quedó integrado por 20 afirmaciones. La estructura de seis subescalas se descar tó ya que ningún elemento se vinculó al sexto factor con una carga ma

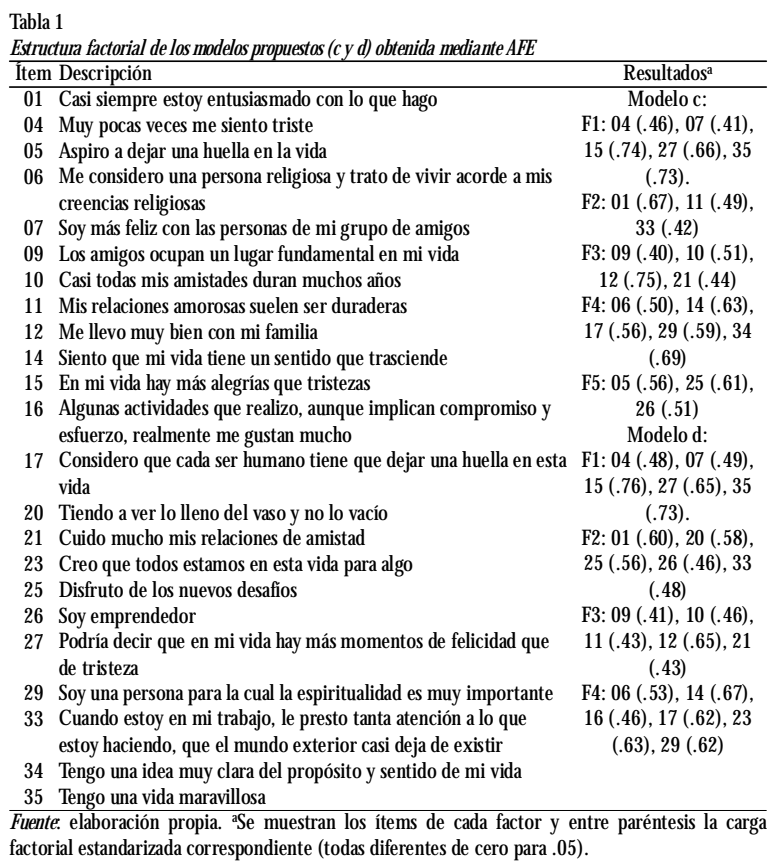




\section{Validez discriminante}

Las razones de las correlaciones HTMT se anexan en la tabla 3. Como puede apreciarse, el modelo pentafactorial de 35 ítems tiene problemas de validez discriminante debido a que varios de los intervalos de confianza incluyen o superan .85. De manera concreta, obsérvese que para la combinación entre el segundo y quinto factor (F2 y F5) la razón es de 1.03 y oscila desde .96 hasta 1.11. Este rango supera al valor referencial previamente señalado, revelando multicolinealidad entre las variables latentes de la escala. Lo anterior se repite en los pares sombreados que se muestran en dicha tabla; sin embargo, tal situación no se aprecia en la versión abreviada del instrumento. En esta oportunidad, ninguno de los intervalos de confianza exhibió una amplitud que incluyera o superara la cifra de .85. Apa rentemente, la eliminación de los ítems con baja saturación realizada durante el AFE subsanó esta problemática y mejoró las propiedades del modelo.

\section{Confiabilidad}

La confiabilidad se presenta en la tabla 2 . El modelo b refleja niveles de consistencia interna inaceptables para F2, cuestionables para F3, aceptables paraF1 y F5, buenos para F4 y excelentes parael total. Por otro lado, el modelo c ostentaíndices inaceptables para $F 2$, pobres paraF5, cuestionables para F3, aceptables paraF1 y F4 y buenos para el total (para ahondar sobre el criterio de cal ificación de la consistencia interna remítase a Dunn et al., 2014). En lo referente a la CITC, se considera apropiado un rango quefluctúedesde. 30 hasta. 70 , condición que satisfacen todos los coeficientes de la estructura abreviada, no así algunos de los factores del instrumento original, en los que se identifican correlaciones menores que . 30 en F1, F2, F3 y en el total.

\section{Discusión}

De acuerdo con los resultados expuestos se puede interpretar que la escala examinada para medir el bienestar psicológico diseñada por Soler (2016) replica la estructura factorial basada en la propuesta teórica de Seligman (2011), en la que los cinco factores que definen el bienestar psicológico multidimensional adolescente son las emociones positivas $(\mathrm{P})$, flujo $(\mathrm{E})$, relaciones sociales (R), sentido (M) y logros (A). Es un hecho que plantear modelos en la etapa de exploración fue bastante oportuno para identificar dos propuestas con carácter confirmatorio que se corresponden con la formulación teórica: primero, un modelo pentadimensional con los 35 ítems originales del diseño de Soler; y segundo, un modelo de 20 ítems que se denominó pentadimensional abreviado, en el que los elementos seleccionados saturan en los cinco factores. En consecuencia, y en términos prácticos, se cuenta con dos escalas de diferente longitud.

Asimismo, la versión original y la abreviada fueron examinandas con respecto a la validez convergente con variables que la literatura ha señalado como importantes debido a la relación que guardan con esta (García Álvarez, Soler \& Cobo, 2019; Seligman, 2018). Espećficamente, la escala de bienestar psicológico de Soler se correlacionó de manera positiva con las de autoestima y autoeficacia, mientras que se relacionó de forma negativa o nula con las escalas de malestar psicológico y síntomas depresivos. Igualmente, se asoció de manera positiva y moderada con las puntuaciones de bienestar psicológico multidimensional de Casullo (2002), instrumento tradicionalmente respaldado por la literatura científica latinoamericana. Aunque la asociación encontrada entre las puntuaciones sugiere evidencias adecuadas de validez convergente (M uñiz \& FonsecaPedrero, 2019), la versión abreviada presentó mayores valores en laVME que la versión de 35 items, reflejando mejores atributos en cuanto a esta propiedad.

De igual modo, tanto en la escala original como en la abreviada se examinó la validez discriminante por medio de la razón de las correlaciones HTMT. En este caso, la escala original presentó dificultades al evidenciar multicolinealidad entre las variables Iatentes, aspecto que corrige la versión abreviada de 20 ítems. Esto sugiere que la construcción del bienestar psicológico multidimensional que acá se propone tendría distinción empírica, dando evidencias razonables de validez discriminante (Henseler et al., 2015).

Ahora bien, al examinar la confiabilidad de la escala original se observa que el factor correspondiente a la dimensión de flujo se encuentra en un nivel inaceptable de alrededor de .40, tanto para alfa como para omega. Kern et al. (2015) también reportaron esta dimensión como la de menor consistencia en su estudio. Por otro Iado, la versión abreviada presentó debilidades en el factor de flujo, así como en los de relaciones sociales y logros, con índices de fiabilidad cercanos a .60. Al respecto, incluso Casullo (2002) reportó valores de confiabilidad cercanos a . 50 en cada una de las dimensiones del bienestar desde la perspectiva de Ryff (1989). Esta autora refiere que esto no tendría que ver con la homogeneidad de la escala pues el indicador global es 
apropiado. En tal sentido, cabe destacar que la confiabilidad total de la escala de Soler es adecuada en susdosversiones, aunque los aspectosmencionados constituyen una evidencia que favorece ligeramente al esquema original y significan una oportunidad de mejora para la escala que se sugiere abordar en futuros estudios.

En definitiva, los resultados dan cuenta de la multidimensionalidad del constructo de bienestar psicológico adolescente como lo han expuesto autores que hacen énfasis en la construcción psicosocial de la experiencia subjetiva, en vez de enfocarse en propuestas unidimensionales (C asullo, 2002; García-Álvarez et al., 2020; Kern et al., 2015; Ryff, 2016; Seligman, 2011; Singh \& Raina, 2020). De igual manera, se proponeque la escala original 0 abreviada sea empleada de forma íntegra para evaluar el bienestar psicológico adolescente en conjunto con entrevistas psicológicas; en efecto, se desaconseja emplear las subescal as de estas a modo de factores para medir alguna dimensión de manera aislada ya que el bienestar psicológico es un constructo dimensional que da cuenta de la interrelación de los elementos mencionados en la experiencia adolescente.

Por último, este estudio tiene limitaciones que es importante mencionar. En primer lugar, el muestreo por conveniencia impide obtener una muestra representativa de la población adolescente montevideana, lo que a su vez tiene implicaciones negativas al momento de generalizar los hallazgos. 0 tra de las limitaciones es que en el estudio original la autora evaluó la validez de contenido mediante técnicas cualitativas, sin emplear métodos cuantitativos como el de medir el acuerdo de los evaluadores a través de coeficientes kappa o simila res. Se recomienda que, en futuros estudios y partiendo de esta versión abreviada, se realicen técnicas cuantitativas de validez de contenido con jueces expertos e incluso considerar más evidencias de validez en el diseño de investigación. De igual manera, es interesante que en futuras investigaciones se puedan examinar otras de las propiedades psicométricas de la escala, así como estudiar posibles diferencias por sexo o trayectorias evolutivas y profesionales en la etapa de la adultez.

\section{Conclusiones}

Se concluye que la escala de bienestar multidimensional para adolescentes de Soler en su versión abreviada de 20 ítems cuenta con adecuadas propiedades de validez factorial, confirmando así el modelo teórico PERMA de Seligman. También se concluye que este instrumento cuenta con validez convergente con medidas como las de bienestar psicológico de Casullo, autoestima, autoeficacia, síntomas depresivos y malestar psicológico. Posee además validez discriminante y una confiabilidad total adecuada, siendo estas propieda des psicométricas las que sugieren que es un instrumento válido, breve y confiable para ser administrado en la población adolescente montevideana.

\section{Agradecimientos}

Los autores desean expresar su profundo agradecimiento a cada adolescente, quienes dispusieron solícita mente de su tiempo para participar en el estudio y responder a los instrumentos correspondientes. También desean agradecer al equipo de trabajo de Jóvenes Fuertes por su colaboración y por facilitar el contacto entre los investigadores y las diferentes instituciones educativas de M ontevideo. Sin su ayuda, la culminación de este proyecto no habría sido posible.

\section{Referencias}

Bandalos, D. L. (2014). Relative performance of categorical diagonally weighted least squares and robust maximum likelihood estimation. Structural Equation Modeling: A Multidisciplinary Journal, 21(1), 102-116. doi.org/ 10.1080/ 10705511.2014.859510

Brałen, A., Huta, V., Tyrany, L., \& Thompson, A. (2019). Hedonic and eudaimonic motives toward university studies: How they relate to each other and to wellbeing derived from school. Journal of Positive Psychology andWellbeing,3(2), 179-196.

Brenlla, M. E., \&Aranguren, M. (2010). Adaptación argentinadelaEscaladeM alestar Psicológico deK essler (K10). Revista de Psicología, 28(2),309-340.

Burke, J., \& M inton, S. J. (2019).Well-being in post-primary schools in Ireland: the assessment and contribution of character strengths. Irish Educational Studies, 38(2), 177192.

Butler, J., \& Kern, M. L. (2016). The PERM A-Profiler: A brief multidimensional measure of flourishing. International Journal ofWellbeing, 6(3), 1-48.

Casullo, M. M. (2002). Evaluación del bienestar psicológico. En Casullo, M. M. (Comp.). (2002). Evaluación del bienes tar psicológico en Iberoamérica (pp. 11-29). Buenos Aires: Paidós.

Cobo-Rendón, R., PérezVillalobos, M.V., Hernández, H., \& Aslan Parra, J. (2017). Modelos multidimensionales del bienestar en contextos de Enseñanza Aprendizaje: 
una revisión sistemática. Ecos de la Academia 3(6), 13-28.

DeCarvalho, J., Pereira, N., Pinto, A., \& Maroco, J. (2016). Psychometric Properties of the Mental Health Continuum-ShortForm:A Study of PortugueseSpeaking Children/ Youths. Journal of Child and Family Studies,25 (7), 2141-2154 . doi:10.1007/ s10826-016-0396-7

Demirtaㅇ, A. S. (2020). Cognitive flexibility and mental well-being inTurkish adolescents: The mediating role of academic, socia and emotional self-efficacy. Anales de Psicología/ Annals of Psychology, 36(1), 111-121.

Díaz-Lea, A. C., Blanco Ornelas, L. H., BenitezHernández, Z. P., AguirreVásquez, S. I. \& C Candia Luján, R. (2017). Propiedades psicométricas del Behavioral Regulation in Exercise Q uestionnaire-2 en universitarios mexicanos. Retos, 34(34), 80-84. doi.org/ 10.47197/ retos. v0i34.55794

Diener, E., Emmons, R. A., Larsen, R. J., \& Griffin, S. (1985). Thesatisfaction with lifescale. Journal of Personality Assesment, 49(1), 71-75.

Diener, E., O ishi, S., \&Tay, L. (2018). Advancesin subjective well-being research. Nature Human Behaviour, 2 (4), 253260. doi:10.1038/ s41562-018-0307-6

Disabato, D. J., Goodman, F. R., Kashdan, T. B., Short, J. L., $\&$ Jarden, A. (2016). Different types of well-being? A cross-cultural examination of hedonic and eudaimonic well-being Psychological Assessment, 28(5), 471.

Domínguez-Alonso, J., López-Castedo, A., \& Portela-Pino, I. (2017).Validación del autoinforme de motivos para la práctica del ejercicio físico con adolescentes (AM PEF): diferencias por género, edad y ciclo escolar. Retos, 33(33), 273-278. doi.org/ 10.47197/ retos. V0i33.58963

Dunn,T.J., Baguley, T., \& Brunsden,V. (2014). From al phato omega: A practical solution to the pervasive problem of internal consistency estimation. British Journal of Psychology, 105(3), 399-412. doi.org/ 10.1111/ bjop. 12046

GarcíaÁlvarez, D., Hernández-Ladinde, J., Espinosa-Castro, J. F., \& Soler, M. J. (2020). Salud mental en la adolescencia montevideana: una mirada desde el bienestar psicológico. ArchivosVenezolanos de Farmacologia y Terapéutica, 39 (2), 182-190.

GarcíaÁlvarez, D., Soler, M., y Cobo, R. (2019). Bienestar psicológico en adolescentes: relacionescon autoestima, autoeficacia, malestar psicológico y síntomas depresivos. Revisa de Orientación Educacional, 33(63), 23-43.

Henseler, J., Ringle, C. M., \& Sarstedt, M. (2015). A new criterion for assessing discriminant validity in variancebased structural equation modeling Journal of theAcademy of Marketing Science, 43(1), 115-135. doi.org/ 10.1007/ s11747-014-0403-8

Hernández-Lalinde, J. D., Espinosa-Castro, J. F., Peñaloza
Tarazona, M. E., Rodríguez, J., Chacón-Rangel, J. G., Toloza-Sierra, C. A., Arenas-Torrado, M. K., CarrilloSierra, S. M., Bermúdez-Pirela, V. J. (2018). Sobre el uso adecuado del coeficiente de correlación de Pearson: definición, propiedades y suposiciones ArchivosVenezolanos de Farmacología y Terapéutica, 37(5), 587-595.

Herrero, J., \& M eneses, J. (2006). Short web-based versions of the perceived stress (PSS) and Center for Epidemiological Studies-Depression (CESD) scales: A comparison to pencil and paper responsesamong Internet users. Computers in Human Behavior, 22(5), 830-846. doi.org/ 10.1016/ j.chb.2004.03.007

Hone, L. (2015). Understanding and M esuringWellbeing. (D octor of Philosophy). Auckland University NuevaZelanda. Hone, L., Jarden, A., Schofield, G., \& Duncan, S. (2014). Measuring flourishing: The impact of operational definitions on the prevalence of high levels of wellbeing International Journal ofNel being, 4(1), 62-90. doi:10.5502/ ijw. v4i1.4

Isabel, P. (2016). Problemas de salud mental en la adolescencia:Vulnerabilidad, riesgo y oportunidad. Adolescencia 11,165-206.

Jayawickreme, E., Forgeard, M. J. C., \& Seligman, M. E. P. (2012). The Engine of Well-Being Review of General Psychology, 16(4), 327-342. doi:10.1037/ a0027990

Jongbloed, J. A., Lesley. . (2015). Elucidating the constructs happiness and wellbeing: A mixed-methods approach. International Journal ofWellbeing, 5(3), 1-20. doi:10.5502/ ijw.v5i3.1

Kern, M. L., Benson, L., Steinberg, E. A., \& Steinberg, L. (2016). The EPOCH measure of adolescent well-being Psychological Assessment, 28, 586-597.

Kern, M. L.,Waters, L. E., Adler, A. , \&W hite, M.A. (2015). A multidimensional approach to measuring well-being in students: Application of the PERMA framework. The Journal of Positive Psychology, 10(3), 262-271.

Kern, M. L., Waters, L., W hite, M., \& Adler, A. (2014). Assessing employee wellbeing in schools using a multifaceted approach: Associationswith physical heath, life satisfaction, and professional thriving Psychology, 5, 500-513. doi.org/ 10.4236/ psych. 2014.56060

Kessler, R. \& M roczek, D. (1994). Final version of our nonspecific Psychological Distress Scale. Ann Arbor, MI: Survey Research Center of the Institute for Social Research, University of Michigan.

M ateo, C. M., Hernández, M. P., \& Cabrero, R. S. (2019). El bienestar psicológico en la adolescencia: Variables psicológicas asociadas y predictoras. European Journal of Education and Psychology, 12 (2), 139-151.

Montero, I., \& León, O. G. (2007). A guide for naming 
research studies in Psychology. International Journal of Clinical and $\mathrm{H}$ ealth Psychology, 7 (3), 847-862.

Morrish, L., Rickard, N., Chin, T. C., \& Vella-Brodrick, D. A. (2018). Emotion regulation in adolescent well-being and positive education. Journal of $H$ appiness Studies, 19(5), 1543-1564.

Muñiz, J. \& \& Fonseca-Pedrero, E. (2019). Diez pasos parala construcción de un test. Psicothema, 31(1), 7-16.

Norrish, J. M., W illiams, P., O 'Connor, M., \& Robinson, J. (2013). An applied framework for positive education. International Journal of Well being, 3(2), 147-161. doi:10.5502/ ijw.v3i2.2

0 'Connor, M., Sanson, A. V., Toumbourou, J.W., Norrish, J., \& O Isson, C. A. (2017). Does Positive Mental Health in Adolescence Longitudinally Predict Healthy TransitionsinYoungAdulthood?] ournal of HappinessStudies 18(1), 177-198. doi.org/ 10.1007/ s10902-016-9723-3

O liva-Delgado, A., Antolín-Suárez, L., Pertegal-Vega, M. Á., Ríos-Bermúdez, M., Parrajiménez, Á., HernandoGómez, Á., \& ReinaFlores, M. del C. (2011). Instrumentos para la evaluación de la salud mental y el desarrollo positivo adolescente y los activos que lo promueven. Junta de Andalucía, Consejería de Salud.

Pollard, E. L., \& Lee, P. D. (2003). Child well-being: A systematic review of the literature. Social Indicators Research, 61(1), 59-78.

Radloff, L. S. (1977). The CES-D scale: A self-report depression scale for research in the general population. Applied Psychological M easurement, 1(3), 385-401. doi. org/ 10.1177/ 014662167700100306

Rose, T., Joe, S., Williams, A., Harris, R., Betz, G., \& Stewart-brown, S. (2017). M easuring MentalW Wellbeing Among Adolescents: A Systematic Review of Instruments. Journal of Child and Family Studies, 26(9), 2349-2362. doi.org/ 10.1007/ s10826-017-0754-0

Rosenberg, M. (1965). Society and the Adolescent Self-Image. Princeton University Press.

Ryff, C. D. (1989). Happiness is everything, or is it? Explorationson themeaning of psychological well-being Journal of Personality and Social Psychology, 57(6), 10691081.

Ryff, C. D. (2016). Psychological well-being and health: Past, present and future. Psicologia Della Salute, 20(1), 7-16.

Sagone, E. , DeCaroli, M. E., Falanga, R., \& Indiana, M. L. (2020). Resilience and perceived self-efficacy in lifeskills from early to late adolescence. International Journal of AdolescenceandYouth, 25(1), 882-890.

Sainani, K. L. (2015). Dealing with missing data. PM\&R, 7(9), 990-994. doi.org/ 10.1016/ j.pmrj.2015.07.011

Salanova, M., Schaufeli,W., Martinez, I., \& Breso, E. (2010).
How obstacles and facilitators predict academic performance: the mediating role of study burnout and engagement. Anxiety Stress Coping, 23(1), 53-70. doi:10.1080/ 10615800802609965

Sánchez-Hernández, Ó ., M éndez, F. X.,Ato, M., \& Garber, J. (2019). Prevention of Depressive Symptoms and Promotion ofWell-being inAdolescents: A Randomized Controlled Trial of the Smile Program. Anales de Psicología/ Annals of Psychology, 35(2), 300-313.

Sawyer, S. M., Afifi, R. A., Bearinger, L. H., Blakemore, S.-J., Dick, B., Ezeh, A. C., \& Patton, G. C. (2012). Adolescence: a foundation for future health. The Lancet, 379(9826), 1630-1640.

Schueller, S. M., \& Seligman, M. E. P. (2010). Pursuit of pleasure, engagement, and meaning: Relationships to subjective and objective measures of well-being Journal of Positive Psychology, 5(4), 253-263. doi:10.1080/ 17439761003794130

Schwarzer, R., \& Baessler, J. (1996). Evaluación de la autoeficacia: Adaptación española de la Escala de Autoeficacia general. Ansiedad y Estrés 2(1), 1-8.

Seligman, M. (2011). Flourish: A visionary new understanding of happiness and well-being (1.a ed.). Free Pr.

Seligman, M. (2018). PERMA and the building blocks of well-being The Journal of Positive Psychology, 13(4), 333335

Shinde, V. (2017). H appiness: Hedonic and eudaimonic. Indian Journal of Positive Psychology, 8(2), 169-173.

Singh, K., \& Raina, M. (2020). Demographic Correlates andValidation of PERMA andW EMW BSScalesin Indian Adolescents. Child Indicators Research, 13(4), 1175-1186.

Soler, M. (2016). Virtud del coraje en relación al bienestar psicológico en adolescentes (Tesis Doctoral inédita de la Universidad de Flores). BuenosAires, Argentina.

Trigueros, R., A guilar-Parra, J., González-Santos, J., \& Cangas, A. (2019). Validación y adaptación de la escala de control psicológico del profesor hacia las clases de educación física y su efecto sobre las frustraciones de las necesidadespsicológicasbásicas. Retos, 37(37), 167-173. doi.org/ 10.47197/ retos. v37i37.71550

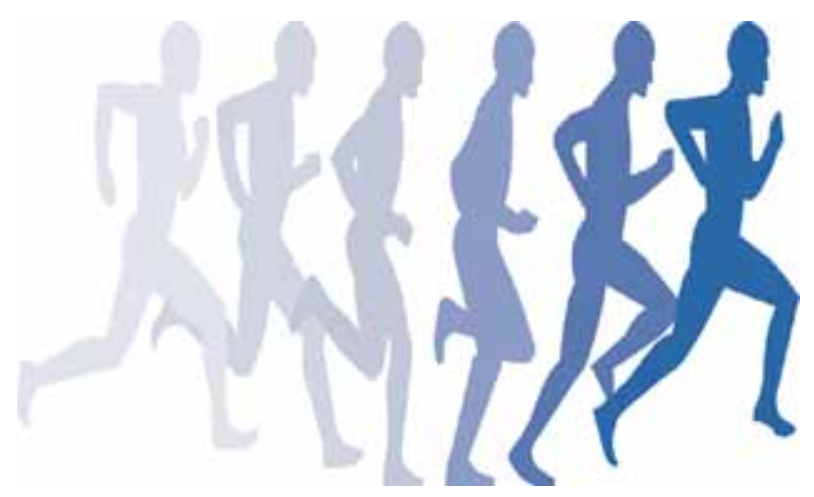

